\section{PTU-115 HEPATITIS B IN PREGNANCY: WHAT HAPPENS TO THE INFANTS?}

doi:10.1136/gutjnl-2013-304907.205

1."J Dyson, ${ }^{2} \mathrm{~J}$ Waller, ${ }^{3} \mathrm{E}$ Michael, ${ }^{3} \mathrm{~A}$ Turley, ${ }^{2,4} \mathrm{~S}$ Moses, ${ }^{2} \mathrm{M}$ Valappil, ${ }^{1,4} \mathrm{M}$ Hudson, ${ }^{1.4} \mathrm{M}$ Bassendine, ${ }^{1,4} \mathrm{~S}$ McPherson. 'Liver Unit, Freeman Hospital; ${ }^{2}$ Health Protection Agency; ${ }^{3}$ Department of Obstetrics, Roval Victoria Infirmary; ${ }^{4}$ nstitute of Cellular Medicine, Newcastle University, Newcastle upon Tyne, UK

Introduction Antenatal screening for Hepatitis B (HBV) has been offered to all pregnant women in the UK since 2000. Immunoprophylaxis of infants is essential to reduce the risk of vertical transmission. It is recommended that HBV vaccination (4 serial doses) be given to all infants born to HBV positive mothers. In addition, Hepatitis B immunoglobulin (HBIG) is recommended for infants of Hepatitis $\mathrm{B}$ e antigen $(\mathrm{HBeAg})$ positive mothers. Infants should have post-vaccination testing between 9-18 months. Hepatitis B surface antigen ( $\mathrm{HBsAg}$ ) negative infants with anti-HBs levels $>10 \mathrm{iu} / \mathrm{ml}$ need no further management. If anti-HBs is $<10 \mathrm{iu} / \mathrm{ml}$ infants should receive a second vaccination series. Our aim was to evaluate the management of infants born to HBV positive mothers.

Methods All HBV positive pregnant women seen in our hospital between January 2008 and November 2011 were identified from an obstetric database. We examined if the infants received the recommended vaccinations, HBIG and post-vaccination testing.

Results From a total of 99 pregnancies data was available for 76 infants. All 15 infants born to $\mathrm{HBeAg}$ positive mothers were given HBIG at birth. 7 mothers had a HBV DNA > 107 IU/ml. 2 were treated with antiviral therapy during pregnancy. 58 (76.3\%) infants received a full vaccination course. Only 35 (54.7\%) of the 64 infants who should have had their post-vaccination status checked to date have had this completed. 34 (97.1\%) had an adequate response to vaccination. One infant (who received 3 vaccinations) is $\mathrm{HBsAg}$ positive with a viral load of $5.4 \times 10^{3} \mathrm{iu} / \mathrm{ml}$. The mother was $\mathrm{HBeAg}$ negative with a viral load of $1.7 \times 10^{3} \mathrm{iu} / \mathrm{ml}$.

Conclusion HBIG was administered appropriately to the infants at highest risk of vertical transmission of HBV. However, completion of the 4 dose HBV vaccination in infants was suboptimal postpartum and post- vaccination testing was inadequate. Efforts to improve this are now in place and include: prospective data collection to improve quality of data; development of a central reminder system to advise family doctors 2 weeks before each vaccination dose is due and ensure this is completed; and introduction of dry blood spot testing of the infants to improve the acceptability of testing.

Disclosure of Interest None Declared

\section{PTU-116 TRANSPLANTATION FOR ALD: LESSONS FROM THE EXPLANT}

doi:10.1136/gutjnl-2013-304907.206

1."J G Brain, ${ }^{2} \mathrm{~S}$ Masson, ${ }^{3} \mathrm{~A}$ Burt. ${ }^{1} \mathrm{ICM}$, Newcastle University; ${ }^{2}$ Liver Unit, Freeman Hospital, Newcastle upon Tyne, UK; ${ }^{3}$ School of Medicine, University of Adelaide, Adelaide, Australia

Introduction Alcoholic liver disease (ALD) remains one of the commonest indications for liver transplantation in Europe. The histological features of ALD vary, depending on extent and stage of injury, No features are reliably pathognomonic of ALD. We describe the histological spectrum of explants of well characterised cohort of patients undergoing transplantation for ALD.

Methods Consecutive explants $(n=84)$ of patients transplanted for ALD in our institution between 2002 and 2011 were selected for retrospective histological assessment. Explants were scored blinded by two pathologists using a predetermined pro-forma. Histological assessment including the presence and degree of cirrhosis, steatosis, inflammation, inclusions, siderosis and neoplastic changes were scored semi-quantitatively.

Results Median age was 54 and the majority (70\%) were male. All patients had a long history of alcohol excess but reported abstinence for at least 6 months by transplantation. The aetiology was ALD $(n=80)$ and mixed ALD/HCV $(n=4)$. The majority $(n=83)$ had a mixed or macronodular cirrhosis with evidence of re-modelling in a significant number; one had pre-septal cirrhosis. Alpha- 1 antitrypsin inclusion bodies were seen in $9(10.7 \%)$; only 4 of these had serum A1AT levels below normal. Parenchymal siderosis was present in 39 (46.4\%); in 19 (22.6\%) this was grade 3-4. Amongst these, only single mutations of the HFE gene were identified. Induced cell change was seen in $67(79.8 \%)$ and $47(56 \%)$ had the "abstinent cell" phenotype. While 46 (54.8\%) had Mallory-denk bodies (MDB), $22(26.1 \%$ of total) patients had both "abstinent cells" and MDB. Ballooning $(\mathrm{n}=45,53.6 \%)$ and steatosis $(\mathrm{n}=31,36.9 \%)$ were also seen. HCC was present in 14 (16.7\%), with dysplastic nodules in $15(17.9 \%)$, small-cell change in $20(23.8 \%)$ and large-cell change $50(59.5 \%)$. Phlebosclerosis and parenchymal extinction were universal findings. Conclusion We describe a wide spectrum of histological features in a large cohort transplanted for end-stage ALD. We demonstrate that despite abstinence, over half have residual MDB and ballooning. Conversely, over half had the recently described "abstinent cell" phenotype. Therefore, the presence of $\mathrm{MDB}$ should not be used as evidence of continued alcohol consumption; the presence of induced or abstinent cells correlates more strongly with reported abstinence.

Disclosure of Interest None Declared

\section{PTU-117 IMAGING HEPATIC NEUTROPHIL MIGRATION WITH INDIUM-111-(111IN)-RADIOLABELLED LEUCOCYTES: A NOVEL NON-INVASIVE DIAGNOSTIC TEST FOR SEVERE ALCOHOLIC HEPATITIS}

doi:10.1136/gutjnl-2013-304907.207

$1,22^{*} \mathrm{~J}$ R Potts, ${ }^{3} \mathrm{M}$ Howard, 'S Verma, ${ }^{2} \mathrm{~A}$ M Peters. ${ }^{1}$ Department of Medicine, Brighton and Sussex Medical School; ${ }^{2}$ Department of Nuclear Medicine; ${ }^{3}$ Department of Histopathology, Brighton and Sussex University Hospitals NHS Trust, Brighton, UK

Introduction Clinical diagnosis of severe alcoholic hepatitis $(\mathrm{SAH})$ can be unreliable and liver biopsy often problematic. Neutrophil infiltration is a key histological feature of SAH and predicts corticosteroid (CS) response (1). We assessed the use of radiolabelled leucocyte scintigraphy to diagnose $\mathrm{SAH}$ through in vivo imaging of hepatic neutrophil migration. Liver signals at ${ }^{111}$ In-leucocyte scintigraphy portray margination at $30 \mathrm{~min}$ post injection (PI) and destruction at $24 \mathrm{~h}$ PI, and are normally matched. We hypothesised that both these functions would be impaired in SAH and liver activity would increase between $30 \mathrm{~min}$ and $24 \mathrm{~h}$ due to neutrophil migration.

Methods 16 patients with SAH (Discriminant Function $54.8 \pm 16.3$, 14 biopsied), 14 with inactive alcoholic cirrhosis (Child-Pugh score $5.4 \pm 0.9$ ) and 11 controls were recruited. Abdominal gamma camera images were obtained $30 \mathrm{~min}$ and $24 \mathrm{~h}$ after IV injection of autologous ${ }^{111}$ In-labelled leucocytes and change in liver activity expressed as a $24 \mathrm{~h}: 30$ min ratio. Biopsies in SAH were stained with the granulocyte marker CD15 and parenchymal neutrophils quantified across 10 high power fields.

Results Liver activity significantly increased in $\mathrm{SAH}$ but was static or fell in cirrhotic and normal control groups (24h:30min liver ratio 2.18 (IOR 1.62) versus $0.97(0.28)$ and $0.78(0.15)$ respectively, $\mathrm{p}<0.001)$. Figure 1 shows example gamma camera images. Liver activity ratios in SAH correlated with histological neutrophil infiltration $(\rho=0.571, p=0.041)$ and microautoradiography demonstrated intact intrahepatic radiolabelled leucocytes as the likely source of $24 \mathrm{~h}$ liver activity. Prominent $30 \mathrm{~min}$ lung activity was a consistent finding in $\mathrm{SAH}$, providing in vivo evidence of neutrophil 
priming. There was no correlation between imaging/histology and $\mathrm{CS}$ response, likely due to the small heterogeneous study group.

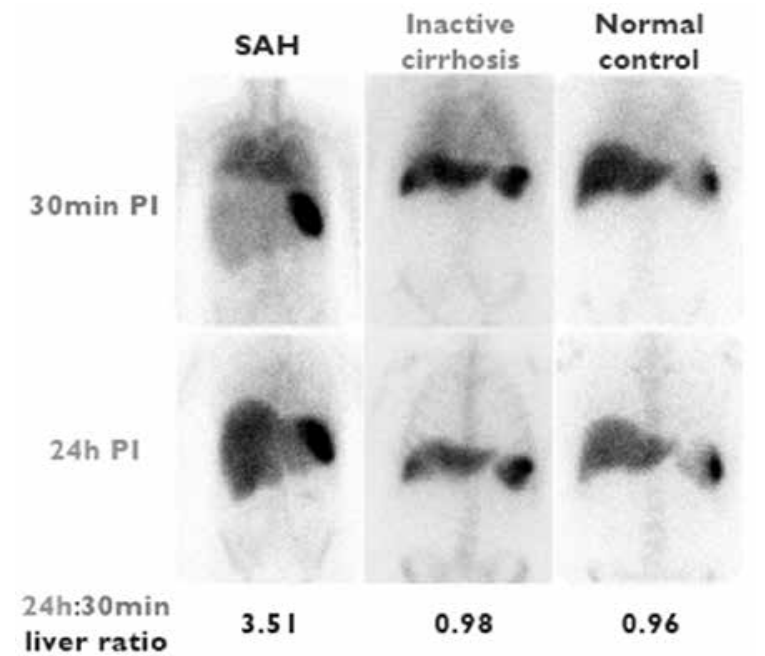

\section{Abstract PTU-117 Figure 1}

Conclusion ${ }^{111}$ In-labelled leucocyte scintigraphy is a novel technique for assessment of hepatic neutrophil migration in SAH. It has potential to be a non-invasive diagnostic tool and may help to prospectively identify those likely to respond to CS.

\section{Disclosure of Interest None Declared}

\section{REFERENCE}

1. Mathurin $\mathrm{P}$, Duchatelle $\mathrm{V}$, Ramond $\mathrm{MJ}$, et al. Survival and prognostic factors in patients with severe alcoholic hepatitis treated with prednisolone. Gastroenterology 1996; 110:1847-53.

\section{PTU-118 LONG-TERM OUTCOME IN SEVERE ALCOHOLIC HEPATITIS}

doi:10.1136/gutjnl-2013-304907.208

1." J R Potts, ${ }^{2}$ S A Goubet, ${ }^{3} \mathrm{M}$ A Heneghan, ' $\mathrm{S}$ Verma. 'Department of Medicine, Brighton and Sussex Medical School; ' $C$ linical Investigation and Research Unit, Brighton and Sussex University Hospitals NHS Trust, Brighton; Institute of Liver Studies, King's College Hospital NHS Foundation Trust, London, UK

Introduction Although short-term outcome in severe alcoholic hepatitis (SAH) is well described, its long-term course is largely unknown. Our aim was to assess long-term outcome in SAH.

Methods Cohort study of patients with SAH (Discriminant Function $\geq 32$ ) admitted to our institute, identified retrospectively 20072009 then prospectively until August 2011. Clinical and laboratory parameters were recorded at accession and subsequent follow-up. Kaplan-Meier (KM) and Cox proportional hazards analyses were performed. Data are presented as mean \pm SD or median (IOR).

Results 109 consecutive patients with SAH were included $(63.3 \%$ men, age $49.6 \pm 9.4 \mathrm{yrs})$ with median follow-up of $40.7 \mathrm{mths}(95 \%$ CI 37.2-44.3). At accession median DF was 58 (34), MELD 23 (6) and Glasgow Alcoholic Hepatitis Score 8 (2). 55.0\% drank spirits, $86.2 \%$ had established cirrhosis and $65.1 \%$ received corticosteroid and/or pentoxifylline therapy. Prevalence of hepatic encephalopathy $(\mathrm{HE})$, infection and hepatorenal syndrome (HRS) were $38.5 \%$, $47.7 \%$ and $18.3 \%$ respectively. Median survival was $22.4 \mathrm{mths}(95 \%$ CI 11.1-33.7), overall mortality being $57.8 \%(n=63)$. All except two deaths were liver related and $33.3 \%$ occurred during the index admission.

In univariate analysis, AST, urea, creatinine, white cell count, and the presence of HE, HRS and infection were statistically significantly associated with mortality ( $p<0.10$ for all), though only HRS was an independent predictor in multivariate analysis (HR 3.842,
95\% CI 2.018-7.312, p < 0.0001). However, all baseline factors were associated with short-term mortality and none predicted death beyond 3 mths.

Of those surviving index hospitalisation ( $\mathrm{n}=87,86$ with available data), 37 (43.0\%) were abstinent at last follow-up. Recidivism occurred in the remaining $49(57.0 \%)$, of whom 33 continued to drink and 16 relapsed after initial abstinence. Abstainers were significantly less likely to require hospital readmission than those with any recidivism (median readmissions/patient 1 vs. $5, p=0.001$ ). In a further univariate analysis, inpatient paracetamol use and abstinence status were associated with mortality after the index hospitalisation ( $p=0.032$ and 0.010 respectively). Only abstinence remained an independent predictor in multivariate analysis (HR 0.402 (95\% CI 0.183-0.883), $\mathrm{p}=0.023$ ). KM analysis showed 3 year survival to be significantly higher in abstainers (75.3\%) compared to relapsed and continued drinkers $(40.2 \%$ and $21.0 \%$ respectively, $\mathrm{p}=0.005)$.

Conclusion Recidivism is common after SAH ( $\sim 65 \%)$ and is the main determinant of the high long-term mortality $(\sim 60 \%)$, which appears unrelated to the severity of the index episode. Novel strategies to improve abstinence following $\mathrm{SAH}$ are urgently needed, especially in view of the increasing numbers of deaths due to alcohol-related liver disease in the UK.

Disclosure of Interest None Declared

\section{PTU-119 HISTOPATHOLOGY AND LONG TERM CLINICAL PROGNOSIS IN HCV. DOES THE BIOPSY STILL HAVE ANYTHING TO ADD?}

doi:10.1136/gutinl-2013-304907.209

1," $\mathrm{M}$ White, ' J John, ${ }^{2,3} \mathrm{R}$ E Swann, ${ }^{2} \mathrm{E}$ Thomson, ${ }^{3} \mathrm{P}$ Mills. 'University of Glasgow Medical School; ${ }^{2}$ MRC University of Glasgow Centre for Virus Research, University of Glasgow: ${ }^{3}$ Gastroenterology, Gatnavel General Hospital, NHS Greater Glasgow And Clyde, Glasgow, UK

Introduction Hepatitis C (HCV) was first characterised in 1989 and most UK sufferers are infected in early adulthood. While a cirrhosis rate of $20 \%$ over 20 years is often quoted, life-long outcomes and factors predicting these are not fully defined. With increasing availability of noninvasive markers of fibrosis, liver biopsy is now rarely performed at diagnosis.

This study aims to explore the value of baseline liver biopsy in determining long-term clinical prognosis.

Methods Patients were identified from a historical HCV study cohort $(n=202)$ at one centre who had a diagnostic liver biopsy at baseline (between 1984 and 2004) and available follow up data. Clinical and histologic data were recorded. Kaplan-Meier plots of time to cirrhosis and multivariate Cox regression were performed.

Results 146 patients had adequate follow up data. The mean duration of follow up from presumed date of infection was 22 (SD 8.51) years with a mean follow up from biopsy of 10 years (SD 5.3). The majority of patients were male $90(62 \%)$ and had been infected through IV drug use 81 (55\%). 58 patients (40\%) had Genotype 1 infection. From baseline biopsies, $44(30 \%)$ had moderate and 12 $(8 \%)$ severe steatosis. 93 (64\%) had Ishak fibrosis scores of 0 or 1 at baseline, 34 (23\%) 2-3 and 10 (7\%) 4-5. 9 patients (6\%) had cirrhosis at baseline.

A total of 31 (21\%) developed clinical cirrhosis during follow up. From these, 11 decompensated and 5 developed hepatocellular carcinoma. Factors associated with shorter time to cirrhosis were later age at diagnosis (HR: 1.08, 95\%CI 1.03-1.13) and increasing Ishak fibrosis score (HR: 2.13 , 95\%CI 1.59-2.85). The 10 year cirrhosis free survival from baseline biopsy was $93 \%$ for Ishak fibrosis scores of 0 or 1 , compared with $78 \%$ and $27 \%$ for scores $2 \& 3$ and $4 \& 5$ respectively (see Fig 1.). 95 (65\%) patients were treated, of whom 64 (67\%) achieved a sustained virological response (SVR) with standard treatment. History of alcohol excess, genotype 1 and severe steatosis were significant negative predictors of SVR. 